\title{
Reducción De La Dbos Y Coliformes Termotolerantes Mediante La Electroquimica En Aguas Servidas Domésticas Del Citrar-Uni-Perú
}

\author{
Agérico Pantoja Cadillo doi , Jocelyn Ambar Gallardo Arismendi, Odón Sánchez Ccoyllo, \\ Edgar Avelino Marcelino Tarmeño \\ Universidad Nacional Tecnológica de Lima Sur, Lima Perú.
}

Recibido: 15/04/2021 Revisado: 21/05/2021 Aceptado: 26/06/2021 Publicado: 28/07/2021

\section{RESUMEN}

Las aguas residuales constituyen un continuo y notable foco de contaminación que perturba el equilibrio natural de suelos y sistemas acuáticos debido a su elevada concentración de contaminantes (materia orgánica, nitrógeno, fósforo, patógenos, metales pesados y constituyentes orgánicos) convirtiéndose así en un riesgo potencial que afecta la salud humana y ambiental.

La presente investigación se centra en determinar la eficiencia de la electrocoagulación en la remoción de la $\mathrm{DBO}_{5}$ y Coliformes Termotolerantes en aguas residuales domésticas del Centro de Investigación de Tratamiento de Aguas Residuales y Residuos Peligrosos (CITRAR). La electrocoagulación es una técnica innovadora en el tratamiento de aguas residuales que consiste en suministrar de corriente eléctrica a electrodos de diversos materiales, en consecuencia, se generan coagulantes debido a la disolución de iones provocando la desestabilización de las partículas contaminantes en un medio acuoso, de tal manera que las fuerzas de atracción predominan sobre las de repulsión facilitando la formación de flóculos más estables que según su densidad pueden sedimentar y/o flotar para su posterior remoción.

La técnica obtuvo eficiencias significativas en la remoción de la $\mathrm{DBO}_{5}$ en $70.782 \%$ y de Coliformes Termotolerantes en $99.630 \%$. Estos resultados se obtuvieron a una densidad de corriente de $45 \mathrm{~A} / \mathrm{m}^{2}$ con un tiempo de tratamiento de 21 minutos.

Palabras claves: electrocoagulación, $\mathrm{DBO}_{5}$, Coliformes Termotolerantes, densidad de corriente, tiempo de tratamiento. 


\begin{abstract}
Wastewater is a continuous and significant source of pollution that disturbs the natural balance of soils and aquatic systems due to its high concentration of pollutants (organic matter, nitrogen, phosphorus, pathogens, heavy metals and organic constituents), thus becoming a potential risk affecting human and environmental health.

This research focuses on determining the efficiency of electrocoagulation in the removal of BOD $_{5}$ and Thermotolerant Coliforms in domestic wastewater from the Center for Research on Wastewater Treatment and Hazardous Waste. Electrocoagulation is an innovative technique in wastewater treatment that consists of supplying electrical current to electrodes of various materials, consequently, coagulants are generated due to the dissolution of ions causing the destabilization of contaminating particles in an aqueous medium, so that the forces of attraction predominate over those of repulsion facilitating the formation of flocs more stable that according to their density can sediment and / or float for subsequent removal. The technique obtained significant efficiencies in the removal of $\mathrm{BOD}_{5}$ in $70.782 \%$ and of Thermotolerant Coliforms in $99.630 \%$. These results were obtained at a current density of 45
\end{abstract}

$\mathrm{A} / \mathrm{m}^{2}$ with a treatment time of 21 minutes.

Key words: electrocoagulation, $\mathrm{BOD}_{5}$, Thermotolerant Coliforms, current density, treatment time. 


\section{INTRODUCCIÓN}

Las Plantas de Tratamiento de Aguas Residuales (PTAR) en el Perú incluye deficiencias en su operatividad, equipamiento y mantenimiento que como consecuencia genera problemas insalubres, aumentando el riesgo de enfermedades debido al elevado contenido de carga orgánica e inorgánica de los efluentes tratados.

Las técnicas de tratamiento más utilizadas para efluentes industriales y domésticos, incluyen procesos biológicos y fisicoquímicos (en el Perú, la tecnología del tratamiento secundario por lagunas facultativas es la más aplicada representando al 75\% de todas las PTAR) (SUNASS, 2016). Sin embargo, existen técnicas innovadoras para el tratamiento de aguas servidas o residuales basados en la electroquímica. Dentro de este grupo se tiene a la electrocoagulación, técnica que consiste en la adición de iones metálicos coagulantes provenientes de un electrodo de sacrificio (ánodo) en un medio acuoso, hecho que facilita la aglomeración de cargas contaminantes para su posterior remoción. La técnica requiere de equipamiento básico y de fácil operación, además posee una alta tasa de efectividad en la remoción de un amplio rango de contaminantes de aguas provenientes de diversos usos.

Hernández (2011) aplicó la técnica en el tratamiento de aguas a la salida de un depurador y aguas que circulan por corrientes fluviales en España; obteniendo eficiencias en la reducción de un 85\% de Sólidos Suspendidos, 92\% de turbidez, 65\% de nitrógeno y 100\% de fósforo, así como la disminución de la contaminación biológica del agua en $99.9 \%$ de E. coli y de coliformes totales en $99.9 \%$, analizando también el consumo energético del proceso. Otra investigación es la de Medina y Peralta (2015), quienes evaluaron un prototipo de electrocoagulación para el tratamiento de aguas residuales provenientes de diferentes actividades industriales con alto contenido de materia orgánica (DBO y DQO) y coloración. Determinaron la remoción de un $87.74 \%$ de unidades de color, $64.07 \%$ de la DBO y $83.21 \%$ de la DQO. Se obtuvo variables de influencia mayor en la eficiencia del equipo básico de electrocoagulación un caudal de 50 $\mathrm{mL} / \mathrm{min}$, el aluminio como los mejores electrodos, un voltaje de $30 \mathrm{~V}$ y una distancia entre electrodos de $1 \mathrm{~cm}$. 
La investigación está basado en la aplicación de la electroquímica-electrocoagulación en el tratamiento de aguas residuales domésticas del CITRAR-UNI, provenientes de aguas residuales domesticas de, con el objetivo de disminuir las concentraciones de la Demanda Bioquímica de Oxígeno ( $\left.\mathrm{DBO}_{5}\right)$ y Coliformes Termotolerantes, determinando las mejores condiciones de tratamiento (densidad de corriente y tiempo de tratamiento) en donde se logrará una mayor eficiencia de remoción.

\section{MATERIALES Y MÉTODOS}

La investigación realizada fue de tipo experimental. Behar Rivero (2008) menciona que, en un estudio experimental, el investigador desea comprobar los efectos de una intervención específica y mantiene un papel activo.

El diseño de la investigación corresponde propuesto Tam Málaga, Vera y Oliveros Ramos (2008). Que considera variables independiente y dependiente.

La población de estudio fue el agua residual doméstica que abastece al CITRAR. El diseño experimental consistió en efectuar 2 réplicas con 9 pruebas cada una. Por cada ensayo se utilizó $1300 \mathrm{~mL}$ de muestra de agua residual doméstica, requiriendo así un volumen total de 23.4 litros sometido a tratamiento.

Las muestras simples se recolectaron de forma puntual en el área de salida del medidor de caudales del CITRAR. En el CITRAR se somete a tratamiento un caudal de $10 \mathrm{~L} / \mathrm{s}$ y es en horas diurnas donde se genera una elevada concentración de la $\mathrm{DBO}_{5}$ y Coliformes Termotolerantes debido a la actividad humana. Es por este motivo que el muestreo se efectuó en horas de la mañana, obteniendo muestras simples representativas del efluente.

Materiales utilizados en la presente investigación: tubos de ensayo con tapa hermética, tubos Durham, botellas de vidrio boca ancha, pipetas serológicas de 1 y $10 \mathrm{~mL}$, auxiliar de pipetas, asa de Kolle con alambre de nicrom, gradillas, cintas indicadoras para esterilización a vapor seco y a vapor húmedo, cucharillas, matraz volumétrico de $50 \mathrm{~mL}$, baguetas de vidrio, pisetas, lunas de reloj, micropipeta, mechero de alcohol, puntas estériles para micropipetas, 
vasos de precipitado de $50 \mathrm{~mL}, 100 \mathrm{~mL}$ y $1000 \mathrm{~mL}$ de capacidad, soporte universal con pinza y nuez, magnetos, 20 botellas de plástico de $1000 \mathrm{~mL}$ de capacidad proporcionado por LABECO para muestreo de la $\mathrm{DBO}_{5}$, jarra de plástico de $1000 \mathrm{~mL}, 9$ reactores tipo batch con capacidad de $1300 \mathrm{~mL}$, cables con pinza cocodrilo, 2 electrodo de aluminio, bandeja para depósito de residuos, cooler, bolsas de gel refrigerante, escobillones para tubos, bidón de 20

L de capacidad, papel tisú, algodón, paños absorbentes, cinta adhesiva, etiquetas adhesivas para rotular y papel Kraft.

Insumos químicos: detergente neutro, hipoclorito de sodio, agua destilada, caldo lauryl sulfato, caldo EC, agua peptona, alcohol isopropílico, alcohol de $96^{\circ}$, tiosulfato de sodio al $10 \%$, solución amortiguadora de $\mathrm{pH} 4, \mathrm{pH} 7$ y $\mathrm{pH} 10$, solución amortiguadora de conductividad eléctrica $1413 \mu \mathrm{S} / \mathrm{cm}$.

Equipos: estufa de esterilización a vapor seco MEMMERT SNE-300, balanza analítica digital GRAM SV, cocina eléctrica MAGEFESA cabina de seguridad biológica BIOBASE BSC1500IIA2-X, refrigeradora WHIRLPOOL, autoclave Pressure Gauge GEMMY SA-

232X, termómetro digital SELECTA, pHmetro portátil ECOTESTR, conductímetro portátil ECOTESTR, agitador magnético VELP SCIENTIFICA, cronómetro digital HERTER, voltímetro, fuente de poder regulable marca PRASEK, agitador vórtex SELECTA, baño maría GEMMYCO YCW-010-E y baño maría SELECTA DIGITERM 100.

Indumentaria de protección personal: guantes de nitrilo y látex, guardapolvo, mascarilla.

\section{Procedimiento}

Limpieza, secado y esterilización de materiales de vidrio: los materiales de vidrio se lavaron con agua potable y detergente, el enguaje final de estos fue con agua destilada para luego secarse en estufa a vapor seco a una temperatura de $150^{\circ} \mathrm{C}$ durante 3 horas, finalmente se envolvieron con papel kraft y se esterilizaron a una temperatura de $170^{\circ} \mathrm{C}$ durante 2 horas.

Preparación de medios de cultivo y agua peptona: el medio Lauril Sulfato, el medio EC y el agua peptona se prepararon según las disposiciones de cada envase (Caldo Lauryl Sulfato,

$35.6 \mathrm{~g}$ en $1000 \mathrm{~mL}$ de agua destilada; caldo EC, $37 \mathrm{~g}$ en $1000 \mathrm{~mL}$ de agua destilada y agua peptona, $25.5 \mathrm{~g}$ en $1000 \mathrm{~mL}$ de agua destilada). La dilución completa se obtuvo sometiendo a calentamiento los medios de cultivo durante $10 \mathrm{~min}$ aproximadamente.

Distribución de caldos y agua peptona: los caldos y agua peptona se distribuyeron en tubos de ensayo (150 tubos con $10 \mathrm{~mL}$ de caldo Lauryl, 150 tubos con $10 \mathrm{~mL}$ de caldo EC y 50 
tubos con $9 \mathrm{~mL}$ de agua peptona) dentro de una cabina de bioseguridad en condiciones asépticas. Los materiales se desinfectaron con alcohol isopropílico antes de introducirse a la cabina

Autoclavado de caldos y agua peptona: el manual de los Métodos para el Análisis de Aguas Potables y Residuales indica que el autoclavado de caldo Lauryl, caldo EC y agua peptona debe efectuarse durante $15 \mathrm{~min}$ a $121^{\circ} \mathrm{C}$. Condiciones que se aplicaron en el autoclavado de los medios, además de utilizar la cinta indicadora para asegurar la eficiente esterilización a vapor húmedo. Finalizado el proceso, los tubos fueron rotulados y conservados en refrigeración hasta su utilización. Cabe indicar que los medios tienen un límite de almacenamiento de 3 meses.

Esterilización de envase para muestra de coliformes termotolerantes: fue necesario agregar $0.2 \mathrm{~mL}$ de $\mathrm{Na}_{2} \mathrm{~S}_{2} \mathrm{O}_{3}$ al $10 \%$ en una botella de vidrio aforada con boca ancha de $250 \mathrm{~mL}$ de capacidad para su esterilización a vapor seco a una temperatura de $170^{\circ} \mathrm{C}$ durante 2 horas; este insumo químico inactiva la acción bactericida del cloro durante el muestreo y el traslado de la muestra al laboratorio.

Toma de muestras de aguas residuales domésticas: la toma de muestras se realizó según las indicaciones contenidas en los Métodos para el Análisis de Aguas Potables y Residuales APHA, AWWA, WEF del año 2005 y la Resolución Ministerial publicada en el peruana $\mathrm{N}^{\circ}$

273-2013.

Medición de parámetros de campo in situ: la medición de temperatura se efectuó con un termómetro digital mientras que la medición de $\mathrm{pH}$ con un pHmetro previamente calibrado con soluciones buffer de pH 4, pH 7 y pH 10, asímismo se llevó a cabo la medición de la conductividad eléctrica con un conductímetro de la marca Ecotestr con previa calibración.

Muestreo de la $\mathrm{DBO}_{5}$ : el muestreo de agua residual doméstica para el análisis de la $\mathrm{DBO}_{5}$ se recolectó en botellas de $1000 \mathrm{~mL}$ de capacidad proporcionadas por LABECO. El envase se saturó hasta hacer rebose, evitando que queden espacios de aire en el interior. La muestra se conservó a $4^{\circ} \mathrm{C}$ y su análisis se efectuó antes de las 48 horas.

Muestreo de coliformes termotolerantes: se recolectó aproximadamente $250 \mathrm{~mL}$ de agua residual doméstica en una botella de vidrio boca ancha, previamente esterilizada con tiosulfato de sodio. La toma de muestra se realizó en contracorriente al flujo, la botella se mantuvo cerrada hasta alcanzar una mínima distancia para la toma de muestra, luego se abrió 
y se recolectó la cantidad suficiente para cerrar inmediatamente. La muestra se conservó a $4^{\circ} \mathrm{C}$ durante su almacenamiento y transporte.

Muestreo de agua residual doméstica para someter a tratamiento de electrocoagulación: por cada réplica del diseño experimental se recolectó aproximadamente 15 litros de agua residual doméstica. El traslado de la muestra hacia el laboratorio de Biología de la UNTELS fue inmediato.

Acondicionamiento del sistema de electrocoagulación: fue necesario diseñar el reactor batch (9 unidades) de material acrílico transparente de dimensiones $9 \mathrm{~cm}$ (ancho) x $9 \mathrm{~cm}$ (largo) x $19 \mathrm{~cm}$ (altura). En la base, se colocó una pieza rectangular de acrílico con ranuras de $10 \mathrm{~mm}$ de separación para el encaje de electrodos de aluminio. Asimismo, se adaptó una válvula de paso de plástico para la salida de muestra. La densidad de corriente $\left(\mathrm{A} / \mathrm{m}^{2}\right)$ suministrada al sistema, lo proporcionó una fuente de amperaje (0-5A) y de voltaje (0-32V) regulable de la marca PRASEK. El paso de corriente a electrodos de aluminio (dimensiones: $7.5 \mathrm{~cm}$ x $15 \mathrm{~cm}$ x $0.8 \mathrm{~cm}$ ) convierte al polo positivo de la fuente en el ánodo o electrodo de sacrificio del sistema donde ocurren los procesos de oxidación (generación del ion $\mathrm{Al}^{3+}$ ); simultáneamente el polo negativo funciona como cátodo, en el cual ocurren los procesos de reducción (conversión de los protones del agua en hidrógeno). Se utilizó también un voltímetro para corroborar el valor del voltaje otorgado al sistema y un cronómetro para controlar el tiempo de tratamiento. Para homogenizar la dosis de coagulante suministrado por el electrodo de sacrificio, fue necesario hacer uso de un agitador magnético. La figura 1 muestra el acondicionamiento del sistema.

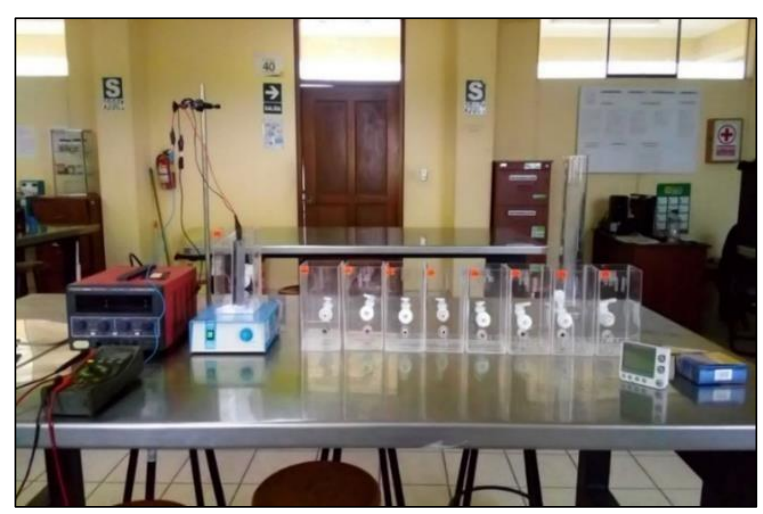

Figura 1. Sistema de electrocoagulación.

Tratamiento de electrocoagulación: previo al tratamiento, se homogenizó el agua residual doméstica para luego descargar al reactor $1300 \mathrm{~mL}$ de muestra. La fuente de amperaje y 
voltaje variable reguló el abastecimiento de energía al sistema con la finalidad de obtener las densidades de corriente establecidas en el diseño experimental (cuadro I). Paralelamente el agitador magnético y el cronómetro se activaron para iniciar el proceso (fig. 2). Finalizado el tratamiento, el sistema fue desmontado para retirar el reactor mientras que los procesos de desestabilización de coloides continuaron, estableciendo un periodo de $40 \mathrm{~min}$ de reposo, tiempo en el que se observó la separación de fases (fig. 3).

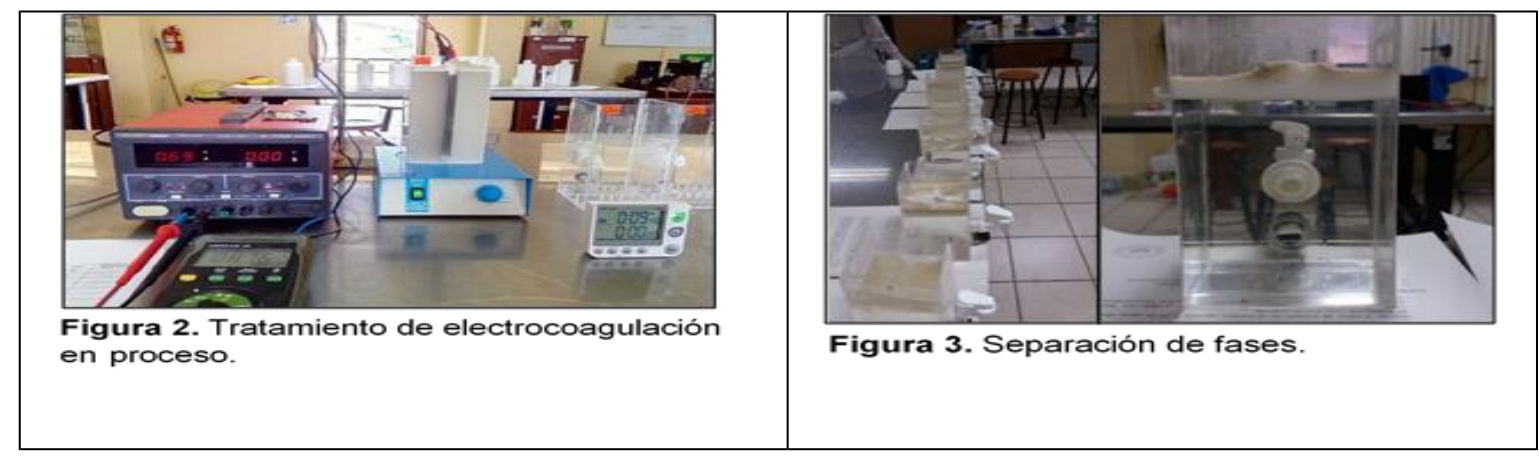

Análisis de coliformes termotolerantes: el análisis para coliformes termotolerantes de la muestra de caracterización y las muestras obtenidas tras el tratamiento, fue el método de recuento por dilución en tubo (Número Más Probable-NMP), procedimiento que se detalla en los Métodos para el Análisis de Aguas Potables y Residuales APHA-AWWA-WEF (2005) en su Parte 9000 Examen Microbiológico de las Aguas. 9221, Técnica de fermentación en tubo múltiple para miembros del grupo de los coliformes. Previo al análisis, los tubos con caldo Lauril y agua peptona se incubaron durante 24 horas a $35^{\circ} \mathrm{C}$ con el propósito de descartar falsos positivos, como resultado, ningún tubo presentó crecimiento bacteriano, hecho que permitió cumplir con las condiciones estériles pertinentes para proseguir con las diluciones y la inoculación de muestra dentro de una cabina de bioseguridad. La figura 4 esquematiza el procedimiento para el análisis de Coliformes Termotolerantes: 


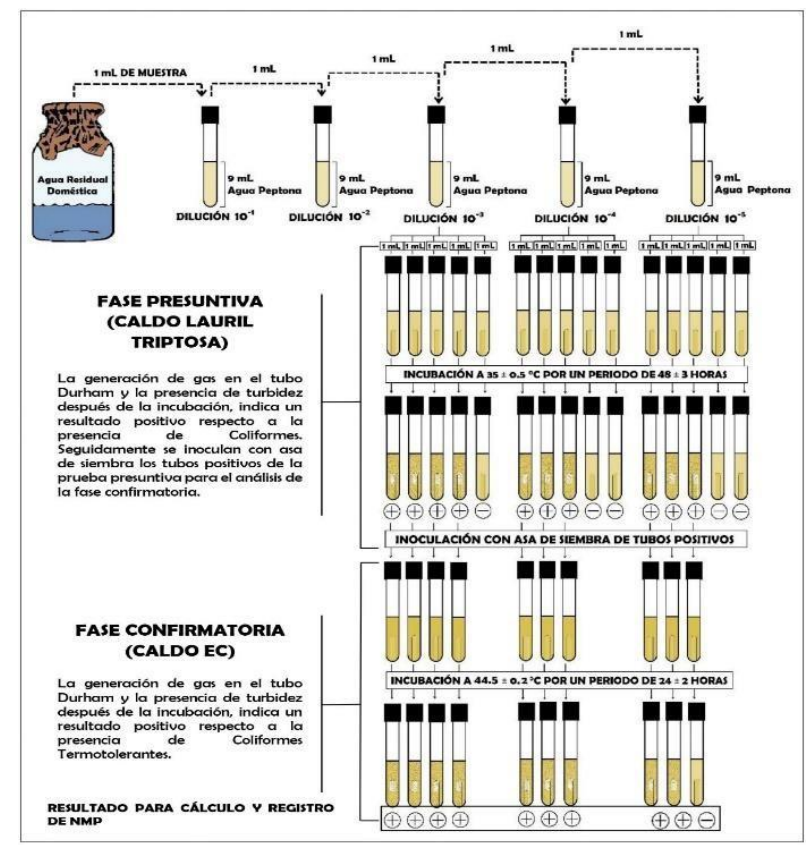

Figura 4. Esquema para el análisis de coliformes termotolerantes

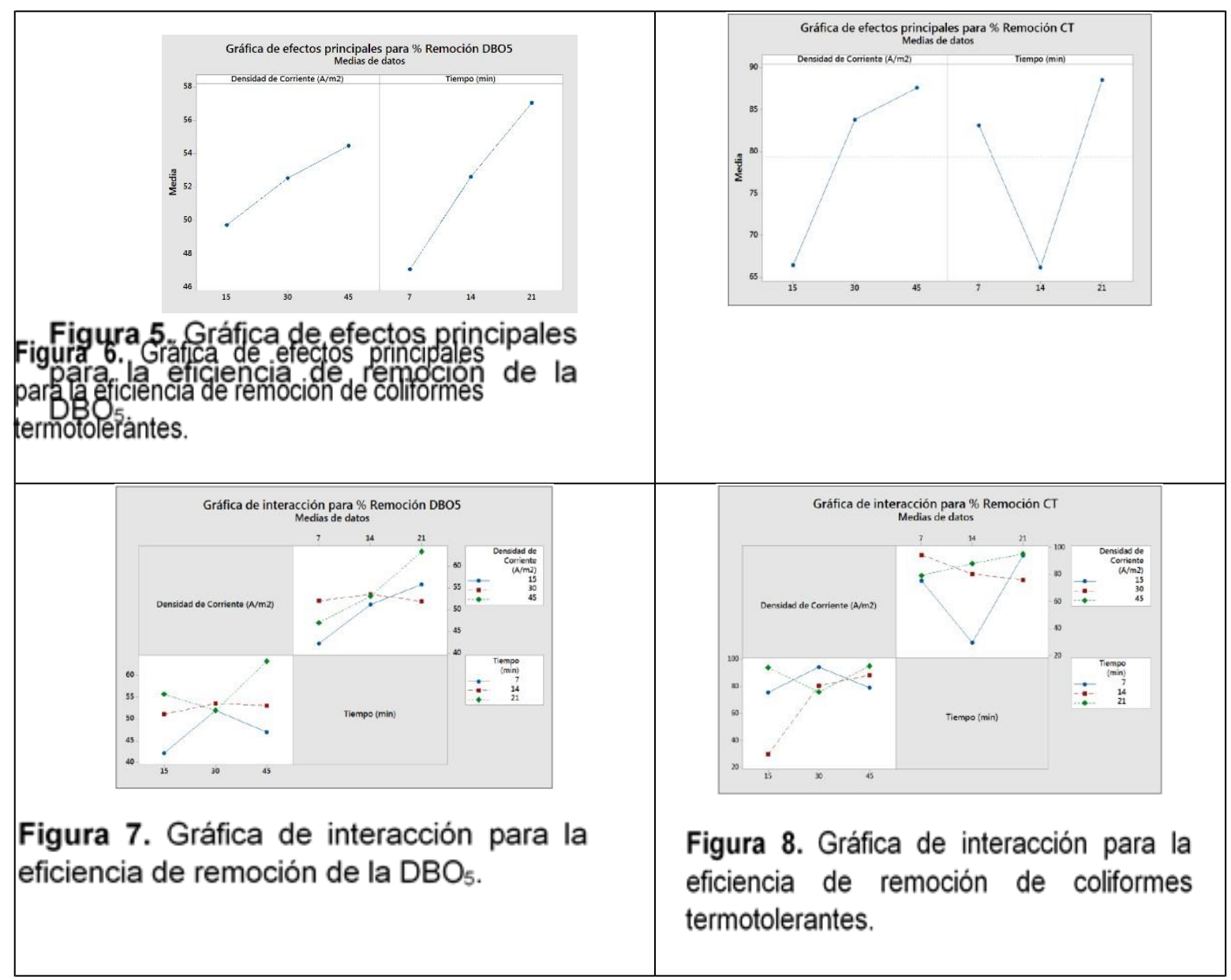

Análisis de la $\mathrm{DBO}_{5}$ : este análisis se determinó mediante la prestación de servicios del Laboratorio de Análisis Ambientales LABECO S.C.R.L; cuyo procedimiento sigue lo 
indicado en los "Métodos para el Análisis de Aguas Potables y Residuales APHA-AWWA- WEF (2012)", específicamente en la parte 5210 B, prueba de DBO de 5 días. Al finalizar cada condición de tratamiento, y al transcurrir el periodo de 40 min de reposo, se efectuó la toma de muestra procedente de la zona clarificada del reactor. Las muestras se conservaron $\mathrm{a} \leq 4^{\circ} \mathrm{C}$ durante su almacenamiento y transporte a LABECO.

\section{Diseño experimental}

Diseño factorial completo general $3^{2}$ : un diseño factorial de 9 pruebas analiza el efecto de 2 o más factores sobre una o varias respuestas mediante la corrida aleatoria de todas las posibles combinaciones que forman los niveles de los factores a analizar, con la finalidad de determinar el mejor desempeño de un proceso (Gutiérrez y de la Vara, 2008).

La elección de los niveles de densidad de corriente y tiempo de tratamiento se establecieron mediante una previa experimentación en el laboratorio de biología de la UNTELS con la finalidad de verificar la efectividad del tratamiento. Los resultados obtenidos indicaron la remoción de un 75\% de la $\mathrm{DBO}_{5}$. Asimismo, los antecedentes de la investigación (Cordero y Manrique, 2009; Barboza, 2011; Pantoja, 2012; Pantoja, 2014; Medina y Peralta, 2015 y Aguilar, 2015) indican una eficiencia significativa en la remoción de los contaminantes que afectan la calidad del agua después de someterse a electrocoagulación en los rangos de 30

$\mathrm{A} / \mathrm{m}^{2}-125 \mathrm{~A} / \mathrm{m}^{2}$ de densidad de corriente y $24 \mathrm{seg}-60 \mathrm{~min}$ de tiempo de tratamiento. En consecuencia, la elección de $15 \mathrm{~A} / \mathrm{m}^{2}, 30 \mathrm{~A} / \mathrm{m}^{2}$ y $45 \mathrm{~A} / \mathrm{m}^{2}$ como niveles de densidad de corriente y $7 \mathrm{~min}, 14 \mathrm{~min}$ y $21 \mathrm{~min}$ como niveles de tiempo de tratamiento permitió analizar la tendencia de las variables respuestas en el presente estudio.

\section{RESULTADOS}

En cuadro I indica caracterización del agua residual doméstica del CITRA

CUADRO I. Resultados de la caracterización del agua residual doméstica del CITRAR

\begin{tabular}{llllcll}
\hline & FECHA & pH & T & CE & DBO $_{5}$ & CT \\
\hline $\begin{array}{l}\text { PRIMERA } \\
\text { CARACTERIZACIÓN }\end{array}$ & $16 / 01 / 18$ & 7.4 & $22.1^{\circ} \mathrm{C}$ & $620 \mu \mathrm{S} / \mathrm{cm}$ & $208.4 \mathrm{mg} / \mathrm{L}$ & $5.4 \times 10^{6} \mathrm{NMP} / 100 \mathrm{~mL}$ \\
\hline $\begin{array}{l}\text { SEGUNDA } \\
\text { CARACTERIZACIÓN }\end{array}$ & $20 / 02 / 18$ & 8.3 & $24.3^{\circ} \mathrm{C}$ & $610 \mu \mathrm{S} / \mathrm{cm}$ & $109.9 \mathrm{mg} / \mathrm{L}$ & $5.4 \times 10^{6} \mathrm{NMP} / 100 \mathrm{~mL}$ \\
\hline \begin{tabular}{l} 
PROMEDIO \\
\hline
\end{tabular} & & 7.85 & $23.2^{\circ} \mathrm{C}$ & $615 \mu \mathrm{S} / \mathrm{cm}$ & $159.15 \mathrm{mg} / \mathrm{L}$ & $5.4 \times 10^{6} \mathrm{NMP} / 100 \mathrm{~mL}$ \\
\hline
\end{tabular}


Resultados del diseño experimental: los resultados (cuadro II) para determinar la eficiencia de remoción de la $\mathrm{DBO}_{5}$ y Coliformes Termotolerantes (CT) se obtuvieron mediante las siguientes fórmulas:

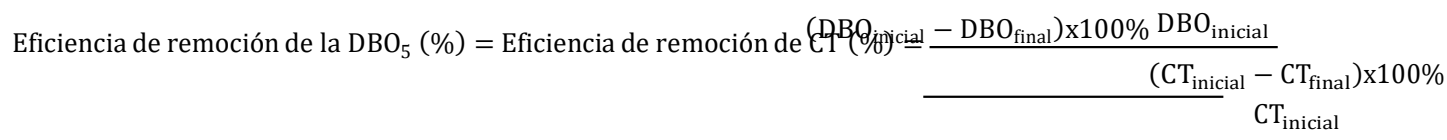

CUADRO II. Resultados del diseño experimental

\begin{tabular}{|c|c|c|c|c|c|c|c|c|}
\hline $\begin{array}{l}\text { ORDEN } \\
\text { ESTADÍST } \\
\text { ICO }\end{array}$ & $\begin{array}{ll}\text { ORD } & \text { I } \\
\text { EN } & 1 \\
\text { DE } & \end{array}$ & $\begin{array}{l}\text { BLOQ } \\
\text { UES } \\
0\end{array}$ & $\begin{array}{l}\text { DENSI } \\
\text { DAD } \\
\text { DE }\end{array}$ & $\begin{array}{r}\text { TIEMPO } \\
\text { DE } \\
\text { TRATA }\end{array}$ & $\begin{array}{l}\text { DBO }_{5} \\
\text { inicial } \\
159.5\end{array}$ & $\begin{array}{l}\text { CT inicial } \\
5.4 \times 10^{6} \\
\text { NMP/100 }\end{array}$ & $\begin{array}{l}\text { \% } \\
\text { REM } \\
\text { OCIO }\end{array}$ & $\begin{array}{l}\% \\
\text { REM } \\
\text { OCIO }\end{array}$ \\
\hline & COR & RÉPLI & CORRI & MIENTO & $\mathrm{mg} / \mathrm{L}$ & $\mathrm{mL}$ & $\mathbf{N}$ & N DE \\
\hline & RIDA & CAS & $\begin{array}{l}\text { ENTE } \\
\left(\mathbf{A} / \mathbf{m}^{2}\right)\end{array}$ & $(\min )$ & $\begin{array}{l}\mathrm{DBO}_{5} \\
(\mathrm{mg} / \mathrm{L})\end{array}$ & $\begin{array}{c}\mathrm{CT} \\
(\mathrm{NMP} / 100 \\
\mathrm{mL})\end{array}$ & $\mathrm{DBO}_{5}$ & CT \\
\hline
\end{tabular}

\begin{tabular}{ccccccccc}
\hline $\mathbf{6}$ & 1 & 1 & 30 & 21 & 88.8 & $4.7 \times 10^{5}$ & 44.204 & 91.296 \\
\hline $\mathbf{7}$ & 2 & 1 & 45 & 7 & 87.2 & $2.1 \times 10^{6}$ & 45.209 & 61.111 \\
\hline $\mathbf{5}$ & 3 & 1 & 30 & 14 & 86.6 & $4 \times 10^{5}$ & 45.586 & 92.593 \\
\hline $\mathbf{3}$ & 4 & 1 & 15 & 21 & 84.5 & $2.1 \times 10^{5}$ & 46.905 & 96.111 \\
\hline $\mathbf{1}$ & 5 & 1 & 15 & 7 & 104.8 & $2.2 \times 10^{6}$ & 34.150 & 59.259 \\
\hline $\mathbf{8}$ & 6 & 1 & 45 & 14 & 79.3 & $1.2 \times 10^{6}$ & 50.173 & 77.778 \\
\hline $\mathbf{4}$ & 7 & 1 & 30 & 7 & 88.7 & $2 \times 10^{5}$ & 44.266 & 96.296 \\
\hline $\mathbf{2}$ & 8 & 1 & 15 & 14 & 81.9 & $2.2 \times 10^{6}$ & 48.539 & 59.259 \\
\hline $\mathbf{9}$ & 9 & 1 & 45 & 21 & 70.0 & $1.5 \times 10^{5}$ & 56.016 & 91.222 \\
\hline $\mathbf{1 1}$ & 10 & 2 & 15 & 14 & 73.5 & $5.4 \times 10^{6}$ & 53.817 & 0.000 \\
\hline $\mathbf{1 6}$ & 11 & 2 & 45 & 7 & 81.5 & $1.4 \times 10^{5}$ & 48.790 & 97.407 \\
\hline $\mathbf{1 8}$ & 12 & 2 & 45 & 21 & 46.5 & $2 \times 10^{4}$ & 70.782 & 99.630 \\
\hline $\mathbf{1 7}$ & 13 & 2 & 45 & 14 & 70.0 & $6.1 \times 10^{4}$ & 56.016 & 98.870 \\
\hline $\mathbf{1 3}$ & 14 & 2 & 30 & 7 & 63.9 & $3.8 \times 10^{5}$ & 59.849 & 92.963 \\
\hline $\mathbf{1 2}$ & 15 & 2 & 15 & 21 & 56.1 & $4.2 \times 10^{5}$ & 64.750 & 92.222 \\
\hline $\mathbf{1 4}$ & 16 & 2 & 30 & 14 & 61.0 & $1.7 \times 10^{6}$ & 61.671 & 68.519 \\
\hline $\mathbf{1 0}$ & 17 & 2 & 15 & 7 & 79.1 & $4.4 \times 10^{5}$ & 50.298 & 91.852 \\
\hline $\mathbf{1 5}$ & 18 & 2 & 30 & 21 & 64.1 & $2.1 \times 10^{6}$ & 59.724 & 61.111 \\
\hline & & & & & & & &
\end{tabular}

\section{DISCUCIÓN}

El análisis de los resultados estadísticos se determinó con un nivel de significancia de $\alpha=0.05$, este valor indica un riesgo de 5\% de concluir que existe una asociación cuando no hay una asociación real.

- Valor $\mathrm{p} \leq \alpha$ : La asociación es estadísticamente significativa.

- Valor $\mathrm{p}>\alpha$ : La asociación no es estadísticamente significativa.

A continuación, se muestra el análisis e interpretación de los resultados experimentales considerando $\alpha=0.05$ : 
Regresión factorial general: \% de remoción de la $\mathrm{DBO}_{5}$ vs densidad de corriente $\left(\mathrm{A} / \mathrm{m}^{2}\right)$ y tiempo (min) analizados en Minitab 17.

La densidad de corriente no influye significativamente en la remoción de la $\mathrm{DBO}_{5}$ en aguas residuales domésticas del CITRAR debido a que no existe asociación estadísticamente significativa entre los factores de prueba y la variable respuesta.

El tiempo de tratamiento influye significativamente en la remoción de la $\mathrm{DBO}_{5}$ en aguas residuales domésticas del CITRAR debido a que exite asociación estadísticamente significativa entre los factores y la variable respuesta.

Regresión factorial general: \% remoción de coliformes termotolerantes vs densidad de corriente $\left(\mathrm{A} / \mathrm{m}^{2}\right)$ y tiempo (min) analizados en Minitab 17.

La densidad de corriente no influye significativamente en la remoción de coliformes termotolerantes en aguas residuales domésticas provenientes del CITRAR debido a que no se comprobó asociación estadísticamente significativa entre los factores y la variable respuesta.

El tiempo de tratamiento del agua no influye significativamente en la remoción de coliformes termotolerantes en aguas residuales domésticas del CITRAR debido a que no existe asociación estadísticamente significativa entre los factores y la variable respuesta.

Efectos principales para la remoción de la $\mathrm{DBO}_{5}$ y coliformes termotolerantes: la figura 5 muestra la gráfica de efectos principales en relación a la eficiencia de remoción de la $\mathrm{DBO}_{5}$, la gráfica indica que la densidad de corriente y el tiempo de tratamiento muestran un efecto lineal sobre la variable respuesta, en este caso sobre la eficiencia de remoción de la $\mathrm{DBO}_{5}$, donde se observa que el mayor porcentaje de remoción (70.782\%) se consigue en las condiciones experimentales máximas ( $45 \mathrm{~A} / \mathrm{m}^{2}$ y $\left.21 \mathrm{~min}\right)$. Asimismo, la figura 6 de efectos principales para la remoción de coliformes termotolerantes indica que, la densidad de corriente tiene un efecto de curvatura apenas perceptible sobre la variable respuesta mientras que el tiempo de tratamiento muestra un efecto de curvatura, en este caso se observa que el mayor porcentaje de remoción $(99.630 \%)$ se consigue en las condiciones experimentales máximas $\left(45 \mathrm{~A} / \mathrm{m}^{2}\right.$ y 21 $\min )$.

Interacciones en la remoción de la $\mathrm{DBO}_{5}$ y coliformes termotolerantes: la figura 7 indica la interacción en cuanto a la eficiencia de remoción de la $\mathrm{DBO}_{5}$, mientras más paralelas se muestren las líneas menor será la dependencia entre la variable respuesta y los factores de la experimentación. La gráfica de interacción evidencia líneas que se intersecan entre sí, hecho que permite afirmar que la eficiencia de remoción de la $\mathrm{DBO}_{5}$ presenta dependencia de los factores de tiempo de tratamiento y densidad de corriente. Asimismo, la figura 8 muestra la 
gráfica de interacción en cuanto a la remoción de coliformes termotolerantes. La gráfica evidencia líneas que se intersecan entre sí, hecho que permite afirmar que la eficiencia de remoción de coliformes termotolerantes presenta dependencia de los factores tiempo de tratamiento y densidad de corriente.

\section{CONCLUSIONES}

Dentro de las conclusiones obtenidas en la presente investigación tenemos que:

La eficiencia del proceso de electroquímica-electrocoagulación en la remoción de la $\mathrm{DBO}_{5}$ y Coliformes Termotolerantes en aguas residuales domésticas del Centro de Investigación en Tratamiento de Aguas Residuales y Residuos Peligrosos (CITRAR) fue de $70.782 \%$ y 99.63\% respectivamente, por lo tanto, la eficiencia de remoción es significativa.

La densidad de corriente que favoreció la remoción de la $\mathrm{DBO}_{5}$ en aguas residuales domésticas del CITRAR mediante electrocoagulación, fue de $45 \mathrm{~A} / \mathrm{m}^{2}$.

El tiempo de tratamiento que favoreció la remoción de la $\mathrm{DBO}_{5}$ en aguas residuales domésticas del CITRAR mediante electrocoagulación, fue de $21 \mathrm{~min}$.

La densidad de corriente que favoreció la remoción de microorganismos, coliformes termo tolerantes en aguas residuales domésticas del CITRAR mediante electrocoagulación, fue de $45 \mathrm{~A} / \mathrm{m}^{2}$.

El tiempo de tratamiento que favoreció la remoción de coliformes termotolerantes en aguas residuales domésticas del CITRAR mediante electrocoagulación, fue de 21 min.

\section{REFERENCIAS BIBLIOGRÁFICAS}

Aguilar Ascon, E. (2015). Evaluación de la Eficiencia de una Celda de Electrocoagulación a escala Laboratorio para el Tratamiento de Agua. (Tesis de Maestría), Lima-Perú.

American Public Health Association [APHA], American Water Works Association [AWWA] y Water Pollution Control Federation [WPCF]. (2005). Métodos Normalizados para el Análisis de Aguas Potables y Residuales. Diaz de Santos, S.A.

Barboza Palomino, G. I. (2011). Reducción de la Carga de Contaminantes de las Aguas Residuales de la Planta de Tratamiento de Totora-Ayacucho Empleando la Técnica 
de Electrocoagulación. (Tesis de Maestría, Universidad Nacional de Ingeniería), LimaPerú.

Behar Rivero, D. S. (2008). Metodología de la Investigación. Shalom. Recuperado el 15 de marzo de 2018, de http://rdigital.unicv.edu.cv/bitstream/123456789/106/3/Libro\%20metodologia\%20i nvestigacion\%20este.pdf

Cordero Medina, E. E., \& Manrique Acosta, J. A. (2009). Evaluación del Efecto de la Electrocoagulación en la Disminución de la Carga Contaminante en Aguas Residuales. (Tesis de Pregrado, Universidad Rafael Urdaneta), Maracaibo- Venezuela.

Gutierréz Pulido, H., \& De la Vara, S. R. (2008). Análisis y diseño de experimentos (Segunda ed.). México D.F.: McGraw-Hill Interamericana.

Hernández Lehmann, P. S. (2011). Investigación sobre Procesos Avanzados de Tratamiento y Depuración de las Aguas mediante Electrocoagulación. (Tesis de Doctorado, Universidad Politécnica de Madrid), Madrid-España.

Irazoque Castañeda, S. (2014). Evaluación de corriente pulsada y otras variables en la remoción de sílice por electrocoagulación. (Tesis de maestría, Universidad Nacional Autónoma de México), México D. F.

Medina Cevallos, J. F., \& Peralta Calderon, M. (2015). Validación de un Prototipo de Electrocoagulación para el Tratamiento de Aguas Residuales de Alta Carga Orgánica presentes en la Industria. (Tesis de Pregrado, Universidad Politécnica Salesiana), Cuenca.

Oficina de Medio Ambiente [OMA]. (24 de abril de 2014). Protocolo de Monitoreo de la Calidad de los Efluentes de las Plantas de Tratamiento de Aguas Residuales Domésticas o Municipales. Ministerio de Vivienda, Construcción y Saneamiento [MVCS], Lima. Recuperado el 25 de marzo de 2017, de file:///C:/Users/Qu\%C3\%ADmica\%20General\%2001/Downloads/1057.pdf.

Pantoja Piarpuzan, E. T. (2012). Aplicación de la Electrocoagulación y Floculación sobre el Tratamiento del Drenaje Ácido Minas de Carbón. (Tesis de Pregrado), Santiago de Cali-Colombia. 
Superintendencia Nacional de Servicios de Saneamiento y Cooperación Alemana de Desarrollo (SUNASS). (2016). Diagnostico de las Plantas de Tratamiento de Aguas Residuales en el Ámbito de Operación de las Entidades Prestadoras de Servicios de Saneamiento. Lima.\|

Tam Málaga, J., Vera, G., \& Oliveros Ramos, R. (2008). Tipos, Métodos y Estrategias de Investigación ciientifica. Pensamiento y áccion.5,145-154 